\title{
Single dose cold blood cardioplegia in adult patients: rationale and technique description
}

\author{
Molina GR*, Correa JR, Rios EG and Abello SJ \\ Department of Cardiovascular Surgery, Hospital Universitario San Ignacio, Bogotá, Colombia
}

\begin{abstract}
Different cardioplegia techniques and strategies have demonstrated to be safe and useful in cardiac surgery. After having employed a series of strategies and according to results published by others and us, ease of use and hospital costs we devised a single dose blood cold cardioplegia strategy. Based on observations and the rationale for the use of blood, crystalloid, cold and warm cardioplegia, we have modified a blood-based cardioplegia to be delivered in a hypothermic single dose strategy with a minimum addition of substrates using each patient's own blood which results in a simple, low cost technique.
\end{abstract}

\section{Introduction}

Currently, "cardioplegia" encompasses various strategies focused on three main goals in most cardiac operations: provide a static, inactive surgical field; static, inactive surgical field; diminish the amount of blood in the operative field and provide "cardiac protection" roughly understood as a steady approaching-to-normal cardiac function after the surgical procedure. This translates fundamentally into absence of myocardial injury markers as seen on acute ischemia with ST segment abnormalities on EKG, cardiac rhythm electrical stability or freedom from arrhythmia (e.g.: VF, AF) and steady ventricular performance under reasonable inotropic support. All different strategies must provide viability of myocytes during ischemia and minimize the damage and adverse effects on cardiac function to a controllable level.

According to their composition there are two streams on cardioplegia; crystalloid cardioplegia, used since the 1960's composed exclusively by crystalloid solutions added with varying types of solute concentrations, ions, chemical buffers and energy precursors in aqueous solutions, showing varying degrees of $\mathrm{pH}$, osmolality and solute concentrations.

Alternatively, blood cardioplegia that has been routinely in use since the 1970's, utilizes blood as a vehicle for added depolarizing, buffering, protective solutions, so a variety of changeable mixtures according to composition, temperature, energy substrates, frequency and administration route, are currently in use [1].

Despite their multiplicity, none of the various methods and strategies of cardioplegia can claim a significant differential advantage over others in clinical grounds. Some studies depict favorable results and describe theoretical advantages and improved outcomes based on a diversity of biochemical markers for ischemia and cellular injury. Study design with diverse methodologies and data analysis of heterogeneous endpoints account for the most frequent and important limitations to obtain proper information when comparative trials and meta/analysis have been attempted. This means that there is still controversy over which should be the method of choice for myocardial protection in adults and pediatric patients judged by clinical outcomes and by other determinants of myocardial function and cell damage [2]. Cardiac surgery centres around the world with large caseloads, excellent clinical results and solid academic background and have adopted myocardial protection schemes based on their own research and their use has spread to other centres with their own modifications.

The debate between crystalloid vs. blood based cardioplegia strategies was approached by a classic meta-analysis conducted in 1987 by the Toronto General Hospital group including 34 trials and showed better results in terms of lower rates of low output syndrome and early CKMB increase using blood cardioplegia but those benefits could not be related to similar incidence on MI or death among patients receiving blood or crystalloid cardioplegia [3]. A later collaborative GermanChinese meta-analysis from 2010, the debate regarding cold vs. warm cardioplegia was analyzed in a study that included 41 randomized controlled trials comparing various warm and cold cardioplegia strategies. That study could not show statistically significant differences in the incidences of clinical events such as risk of in-hospital death, myocardial infarction, low output syndrome, use of IABP and atrial fibrillation between these two groups but again ample methodological disparities occurred [4]. Similar results were obtained in pediatric patients when analyzed by the same group [5].

Cardioplegia strategies based on crystalloid solutions and additional substrates are simple and effective methods for myocardial protection since they show faster availability, less demanding manipulation, theoretical more homogeneous distribution in areas with coronary obstruction due to a better rheology; besides lacking leukocytes could contribute to less reperfusion induced damage. However, crystalloid cardioplegia certainly generates greater haemodilution, requires adding pharmacological substrates necessary to achieve normal enzymatic functions, to obtain appropriate buffering capacity and to

Correspondence to: Germán Molina, Department of Cardiovascular Surgery, Hospital Universitario San Ignacion, Carrera 7 N. 40-62 Bogotá, Colombia, Tel: +573003331660; E-mail: gmolina@husi.org.co

Key words: blood cardioplegia, crystalloid cardioplegia

Received: January 18, 2018; Accepted: February 05, 2018; Published: February 08,2018 
provide availability of energy precursors to power enzymatic cycles. All these additives might generate uncertain effects on cell physiology and are not normally found in human blood.

What can be inferred from meta-analysis is that there are no major clinical outcome differences between blood vs. crystalloid and warm vs. cold cardioplegic strategies; it is also safe to state that there is a tendency towards more favorable outcomes in terms on metabolic performance and myocyte preservation determinants with blood cardioplegia and particularly with continuous warm blood strategies. Continuous warm blood cardioplegic strategies may result in a very complex operative field set up and inadequate when a bloodless field may be required. Pure crystalloid cardioplegic strategies are simple, easy to use but end up in more hemodilution, which will require further interventions.

\section{Rationale}

For more than two decades we have utilized well-established cardioplegia strategies including blood based and crystalloid based solutions with favorable clinical results. Initially we standardized blood cardioplegia with warm induction and repeated doses in 20-minute intervals given in a manual, pressure controlled antegrade mode, infused by the anesthesiologist. Although this is a low-cost strategy it implies contamination risks and requires interrupting the procedure often at difficult cardioplegia delivery situations. Alternatively, Bretschneider's solution was used at our institution during the last decade and became more frequently used than blood cardioplegia in most routine cases and always in emergency operations, neonatal / pediatric surgery, and complex procedures. Although it provided a clear ease of use, higher hemodilution, positive fluid balances and lower hemoglobin concentrations in the early postoperative period were continuously observed.

We planned a cardioplegia strategy to be delivered as a single dose with a simple composition to result in minimum hemodilution and a limited positive fluid balance. In that sense, each patient's own blood derived from the perfusion circuitry with a minimal red cell and coagulation components loss results adequate to provide a vehicle to deliver a solution with minimum of required added elements to obtain normal rheological and osmotic properties. Potassium chloride as a membrane-depolarizing element was to be added to achieve a final concentration of $20-24 \mathrm{mEq} / \mathrm{L}$ similar to most potassium based cardioplegic solutions. Since varying degrees of cellular lactic acidosis are to be expected, sodium bicarbonate was used as an additive to the blood's own buffering systems. All bloods buffering and other rheological properties would not be close to ideal if the final blood dilution resulted inadequately high, so we chose a final 4:1 blood to crystalloid ratio proportion using Ringer's Lactate added potassium chloride as depolarizing agent and sodium bicarbonate as a buffering additive to achieve a final concentration of $25 \mathrm{mEq} / \mathrm{L}$. The 4:1 proportion resulted as tubing and pump conditions allowed this as the most frequent and convenient configuration without mayor adjustments in our set up.

Infusion time of the cardioplegic solution turns out to be a key variable since one of the important factors in Bretschneider group research observations was to deliver a single dose at a slow infusion enough to obtain a homogeneous tissue temperature and extracellular distribution. Studies carried out by his group were supported by histological and metabolic findings at specific conditions of temperature, flow, pressure and time [6,7]. According to recommendations the Bretschneider HTK solution must be infused in a single-dose manner over $8-10 \mathrm{~min}$ requiring a volume of $500-800 \mathrm{cc}$ in canine models. His group also formulated the concept of practical resuscitation time or $\mathrm{t}$-ATP as the time required form myocardial ATP to fall from about $6 \mathrm{mmol} / \mathrm{g}$ wet weight to $4 \mathrm{mmol} / \mathrm{g}$ wet weight. This level was shown to be critical for functional recovery from ischemia and rapid weaning from $\mathrm{CPB}$ [8[]. Similar infusion variables regarding temperature, flow, pressure and time were to be used with the cold 4:1 blood crystalloid solution.

\section{Composition}

Table 1 shows the composition of this single dose blood cardioplegia strategy. The final solution is a $4: 1$ blood to crystalloid mixture. The crystalloid solution is obtained by adding $40 \mathrm{cc}$ of $10 \%$ Potassium chloride ( $80 \mathrm{mEq}$ ) and $30 \mathrm{cc}$ of $10 \% \mathrm{NaHCO} 3(30 \mathrm{mEq})$ to a 1L Ringer's Lactate bag conaining $930 \mathrm{cc}$ of the original solution.

\section{Delivery technique}

Based on Bretschneider's group findings we planned to deliver blood cardioplegia under known volume, flow velocity, time and pressure variables. The total volume for an average adult Bretschneider's cardioplegia during a six to eight-minute infusion varies between 900-1200 cc. Therefore, we calibrated a double tubing roller pump to deliver $1 \mathrm{~L}$ of the blood-crystalloid mixture during six minutes at a flow rate of approximately $160-170 \mathrm{ml} / \mathrm{min}$. Using tubing of $1 / 4 \mathrm{in}$. for blood and 1/16 in. for the crystalloid mixture into the same roller pump gives exactly a final 4:1 proportion. Additionally, we aimed to maintain similar pressure readings as those measured during routine Bretschneider HTK recommended six-minute infusion when infused by $4: 1$ double tubing by a roller pump. The measured pressure at the end of the tubing during Bretschneider cardioplegia infusion was 35-55 mmHg. The distal tubing pressure during pump infusion for adult patients with described setup revealed a measurement between 40-60 mmHg.

The crystalloid mixture bag and blood source line from the oxygenator reservoir are paralleled and fitted into a roller pump, once passed it a coupling Y connector that will deliver it into a single $1 / 4 \mathrm{in}$. tube that goes to the inlet of the MYOtherm ${ }^{\star}$ (Medtronic, Minneapolis, $\mathrm{MN}$ ) cardioplegia heat exchanger (Figure 1). The outflow line from the heat exchanger connects with sterile double Luer-lock connector $1 / 4$ in. tubing that comes from the operative field (Figure 2). The system

Table 1. Composition of blood cardioplegia

\begin{tabular}{|c|c|c|c|}
\hline \multirow{2}{*}{ Blood: 4} & \multicolumn{2}{|l|}{ Solution: 1} & \multirow{2}{*}{ Total composition } \\
\hline & Ringer's lactate & Addition & \\
\hline Potassium: $3.5-5 \mathrm{mEq} / \mathrm{L}$ & Potassium $4 \mathrm{mEq} / \mathrm{L}$ & Potassium Chloride: $80 \mathrm{mEq}$ & $\sim 20 \mathrm{mEq} / \mathrm{L}$ \\
\hline Sodium: $135-145 \mathrm{mEq} / \mathrm{L}$ & Sodium $130 \mathrm{mEq} / \mathrm{L}$ & & $\sim 138 \mathrm{mEq} / \mathrm{L}$ \\
\hline Bicarbonate: $22-28 \mathrm{mEq} / \mathrm{L}$ & & Sodium bicarbonate: $30 \mathrm{mEq}$ & $\sim 23.6 \mathrm{mEq} / \mathrm{L}$ \\
\hline Calcium: $8.5-10.5 \mathrm{mg} / \mathrm{dl}$ & Calcium $3 \mathrm{mEq} / \mathrm{L}$ & & $\sim 7.8 \mathrm{mEq} / \mathrm{L}$ \\
\hline \multicolumn{4}{|l|}{ Phosphorus: $2.7-4.7 \mathrm{mg} / \mathrm{dl}$} \\
\hline \multicolumn{4}{|l|}{ Protein: $6.4-8.3 \mathrm{gr} / \mathrm{dl}$} \\
\hline Albumin: $3.5-5 \mathrm{gr} / \mathrm{dl}$ & & & \\
\hline
\end{tabular}




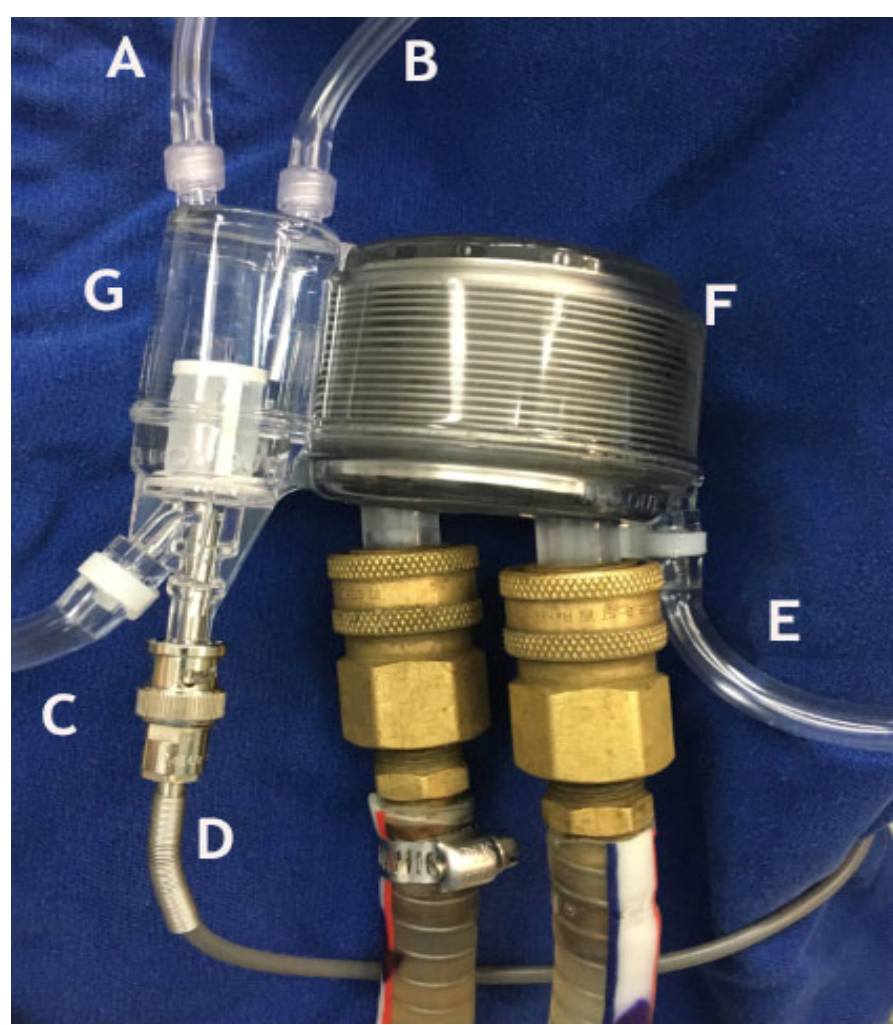

Figure 1. Cardioplegia heat exchanger

Venting Line, B. Pressure Monitoring Line, C. System Outlet, D. Thermometer, E. System Inlet, F. Temperature Exchanger, G. Air Trap/Filter.

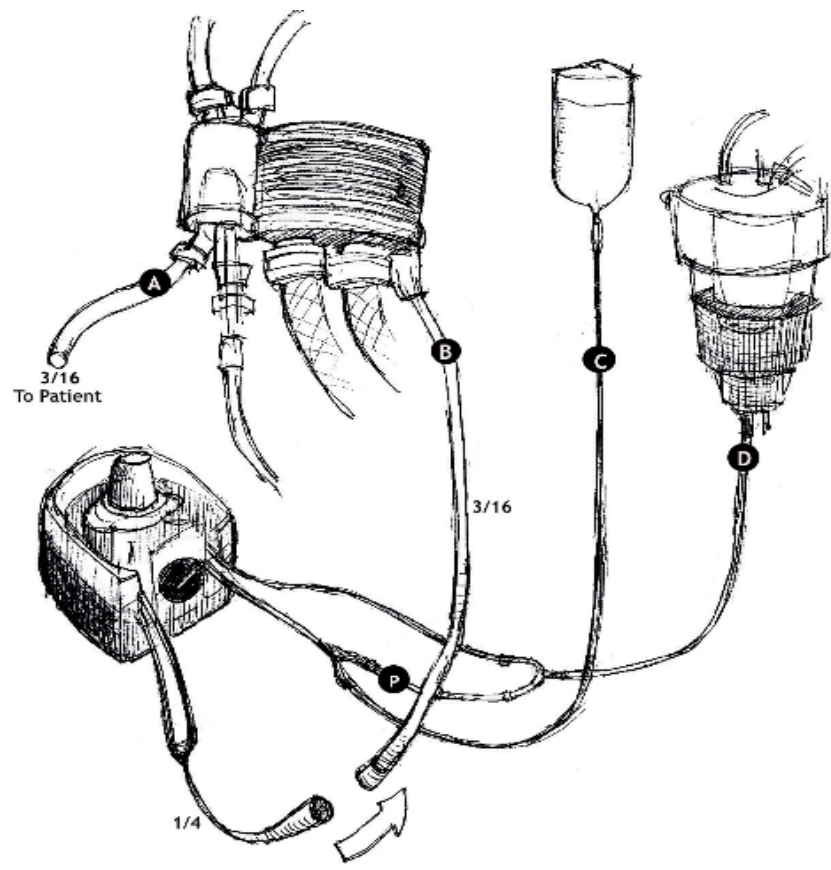

Figure 2. Single dose blood cold cardioplegia setup

A. Outlet line, B. Inlet line, C. Crystalloid Line, D. Blood Line P. Priming connector

priming is done before aortic cross clamping to ensure appropriate mixture delivery. A total volume of approximately $1 \mathrm{Lt}$ is given over a six to eight-minute time span utilizing conventional aortic root cardioplegia needles. In cases with severe aortic insufficiency, sequential direct ostial infusion with hand held cannulas is used starting with the dominant coronary. Infusion time at each ostium is liberally split to obtain similar equivalent times unless a significant size discrepancy is observed between ostia or anatomical abnormalities urge for a different approach.

Other aspects of myocardial preservation should be observed such as preventing LV distension and avoiding rewarming of the heart. Continuous topical cooling with iced saline solution or slush is advisable.

Once cross clamp is applied, and infusion has begun, typical EKG changes can be observed in one to two minutes time and an isoelectric EKG recording is obtained after 3 to 4 minutes that lasts for at least 90 minutes or longer. In long cross clamp time cases repeated maneuvers for topical cooling are prompted. Unless failure in aortic cross clamping occurs no repeated cardioplegia doses are necessary.

\section{Discussion}

Blood and crystalloid cardioplegia strategies have been described many years ago and both have proven their safety and efficacy. Blood based cardioplegia and its modifications include different dosages: induction, maintenance and reperfusion at time intervals of at least 20 minutes. Although blood based cardioplegia makes it less prone to hemodilution, it must maintain a significant proportion of this component. Described blood containing methods are complex and repeated doses are cumbersome. Crystalloid cardioplegia strategies although simple, safe and efficient, produce hemodilution. Methods described with inverse 1:4 blood to crystalloid ratio, call for a greater hemodilution and their circuitry and complex formula make this technique less practical. The proposed dual cardioplegia methods (Buckberg-Del Nido or Buckberg-Beyersdorf) result in a still more complex procedure that needs to cell saver and hemofiltration strategies to avoid hemodilution or multiple dose delivery $[9,10]$.

Our modifications to cold blood cardioplegia aim for a simple, safe and reliable single dose method of operative myocardial protection that can be used in routine and complex cases as well as in minimally invasive procedures. Its methodology is based on observations made by different researchers on composition and delivery techniques. These modifications relate fundamentally to composition, dose and delivery conditions to achieve a thorough and homogeneous distribution of the solution.

\section{Declaration of conflicting interest}

The authors declare there is no conflict of interest.

\section{Funding}

This research received no specific grant from any funding agency in the public, commercial, or not-for-profit sectors.

\section{References}

1. Geissler HJ, Melnhorn U (2006) Cold Crystalloid Cardioplegia. Multimed Man Cardiothorac Surg 109: 1-4. [Crossref]

2. Yau TM, Weisel RD, Mickle DA, Komeda M, Ivanov J, et al. (1992) Alternative techniques of cardioplegia. Circulation 86: II377-II84. [Crossref]

3. Guru V, Omura J, Alghamadi AA, Weisel R, Fremes SE (2006) Is Blood Superior to Crystalloid Cardioplegia? A Meta-Analysis of Randomized Clinical Trials. Circulation 114: I331-I338. [Crossref]

4. Fan Y, Zhang AM, Xiao YB, Weng, YG, Hetzer, R (2010) Warm versus cold cardioplegia for heart surgery: a meta-Analysis. Eur J Cardiothorac Surg 37: 912-919. [Crossef]

5. Fang Y, Long C, Lou S, Guan Y, Fu Z (2015) Blood versus crystalloid cardioplegia for pediatric cardiac surgery: a meta-analysis. Perfusion 30: 529-536. [Crossref]

6. Bretschneider HJ, Hubner G, Knoll D, Lohr B, Nordbeck H, et al. (1975) Myocardia resistance and tolerance to ischemia: physiological and biochemical basis. J Cardiovasc Surg (Torino) 16: 241-260. [Crossref] 
7. Preusse CJ, Bretschneider HJ, Gebhard MM (1988) Myocardial equilibration procedures during cardioplegic coronary perfusion. In Proceedings Symposium Cardioplegia: The First Quarter Century, London 97-106.

8. Gunnes S, Jynge P (2011) Fundamentals of the Past: Cardioplegia: The First Period Revisited. 2011, In New Solutions for the Heart, Podesser BK, Chambers DJ, Editors. Springer Vienna, 15-40
9. Kim K, Ball C, Grady P, Mick S (2014) Use of del Nido Cardioplegia for Adult Cardiac Surgery at the Cleveland Clinic: Perfusion Implications. $J$ Extra Corpor Technol 46: 317-323. [Crossref]

10. Martin J, Benk C (2006) Blood Cardioplegia. Multimed Man Cardiothorac Surg 2006: 1-7. [Crossref]

Copyright: (C2018 Molina GR. This is an open-access article distributed under the terms of the Creative Commons Attribution License, which permits unrestricted use, distribution, and reproduction in any medium, provided the original author and source are credited. 